\title{
Knowledge Acquisition for Fuzzy Expert Systems
}

\author{
Gwo-Jen Hwang \\ Computer Center, National Chiao Tung University, Hsinchu, 300, \\ Taiwan, Republic of China
}

\begin{abstract}
Expert systems have been successfully applied to a wide variety of application domains. To achieve better performance, researchers have tried to employ fuzzy logic to the development of expert systems. However, as fuzzy rules and membership functions are difficult to define, most of the existing tools and environments for expert systems do not support fuzzy representation and reasoning. Thus, it is time-consuming to develop fuzzy expert systems. In this article we propose a new approach to elicit expertise and to generate knowledge bases for fuzzy expert systems. A knowledge acquisition system based upon the approach is also presented, which can help knowledge engineers to create, adjust, debug, and execute fuzzy expert systems. Some control techniques are employed in the knowledge acquisition system so that the concepts of fuzzy logic could be directly applied to conventional expert system shells; moreover, a graphic user interface is provided to facilitate the adjustment of membership functions and the display of outputs. The knowledge acquisition system has been integrated with a popular expert system shell, CLIPS, to offer a complete development environment for knowledge engineers. With the help of this environment, the development of fuzzy expert systems becomes much more convenient and efficient. (1) 1995 John Wiley \& Sons, Inc.
\end{abstract}

\section{INTRODUCTION}

In recent years, expert systems have been applied to a wide variety of application domains, which not only showed the utility of this approach, but also revealed some problems of applying it. Part of the problems in employing conventional expert systems may be due to the use of binary logic to express domain knowledge, whose expressions may not be natural or straightforward and, hence, the expertise is not completely represented. For example, there are only two possible sides (true or false) in binary logic and a clear boundary must be indicated for making choices, while in the real world, it is difficult to judge things to be absolutely true or false. The problem becomes more complicated if linguistic hedges (e.g., very, gradually, moderately, more or less, etc.) are used in expressing expertise. ${ }^{1}$

A good solution to this problem is to employ the concept of Fuzzy Set and Fuzzy Logic in expert systems; that is, fuzzy expert systems. For example, let $E$ represent the set of "the people who are tall." In conventional binary logic, 
we must clearly specify whether an element belongs to $E$ or not. However, in fuzzy logic, each element belongs to the set with a degree of membership. A person of $160-\mathrm{cm}$ height belongs to $E$ with the degree of membership 0.0 ; while the persons of $170-\mathrm{cm}, 175-\mathrm{cm}$, and $180-\mathrm{cm}$ heights may have degrees of membership $0.5,0.75$ and 1.0, respectively. Moreover, when considering the linguistic hedges (e.g., very, gradually, moderately, more or less, etc.), all we need to do is to compute the degrees of memberships according to some hedge functions. For example, let the $F_{v e r}(x)=x^{2}$, where $x$ is degree of memberships without qualifier. If a person is said to be TALL with degree 0.8 , according to $F_{\text {very }}(x)$, we conclude that the person is VERY TALL with degree 0.64 .

However, the development of a fuzzy expert system is time-consuming. The difficulty may be due to the lack of methodologies and tools to help in eliciting knowledge for constructing fuzzy rules and in defining the membership functions for fuzzy values. Moreover, most existing expert system shells do not support fuzzy reasoning, which increases the cost needed for developing fuzzy expert systems. To cope with these problems, we propose a knowledge acquisition system that not only elicits knowledge from domain experts, but also generates knowledge bases for fuzzy expert systems. We have integrated the environment with a popular expert system shell, CLIPS, to enable fuzzy reasoning. Some experimental results showed that the approach is desirable and is worth further study.

In this article, we propose a knowledge acquisition environment that consists of a knowledge acquisition unit, a graphic membership function developer, a fuzzy interface for expert systems shells, and a set of control rules for fuzzy reasoning. The environment has been integrated with a well-known expert system shell to perform fuzzy reasoning. Finally, some test results of an experiment on a practical medical diagnosis problem are shown to evaluate the new approach, and a conclusion is given.

\section{KNOWLEDGE ACQUISITION FOR FUZZY EXPERT SYSTEMS}

One critical bottleneck to building expert systems is the elicitation of knowledge from domain experts. Many knowledge engineering tools (e.g., ETS, ${ }^{2}$ NeoETS, ${ }^{3}$ AQUINAS, ${ }^{4}$ MOLE, ${ }^{5}$ NICOD, ${ }^{6}$ ICONKAT, ${ }^{7}$ KSSO, ${ }^{8}$ ASK, SMORE, ${ }^{10} \mathrm{ALT},{ }^{11}$ RuleCon, ${ }^{12} \mathrm{KITTEN},{ }^{13}$ etc.) and interviewing techniques, ${ }^{14.15}$ have been developed to cope with this problem. In this article, we propose a knowledge acquisition environment, Knowledge Acquisition for Fuzzy Expert Systems (KAFES), which is capable of eliciting expertise from domain experts and generating knowledge bases for fuzzy expert systems. The environment is integrated with a well-known expert system shell, CLIPS, to enable fuzzy reasoning. It consists of four components:

(1) Knowledge acquisition unit: a revised repertory grid mechanism ${ }^{16}$ is used to enable the elicitation of fuzzy knowledge.

(2) Graphic membership function developer: a tool to help knowledge engineers adjust membership functions of fuzzy values. 


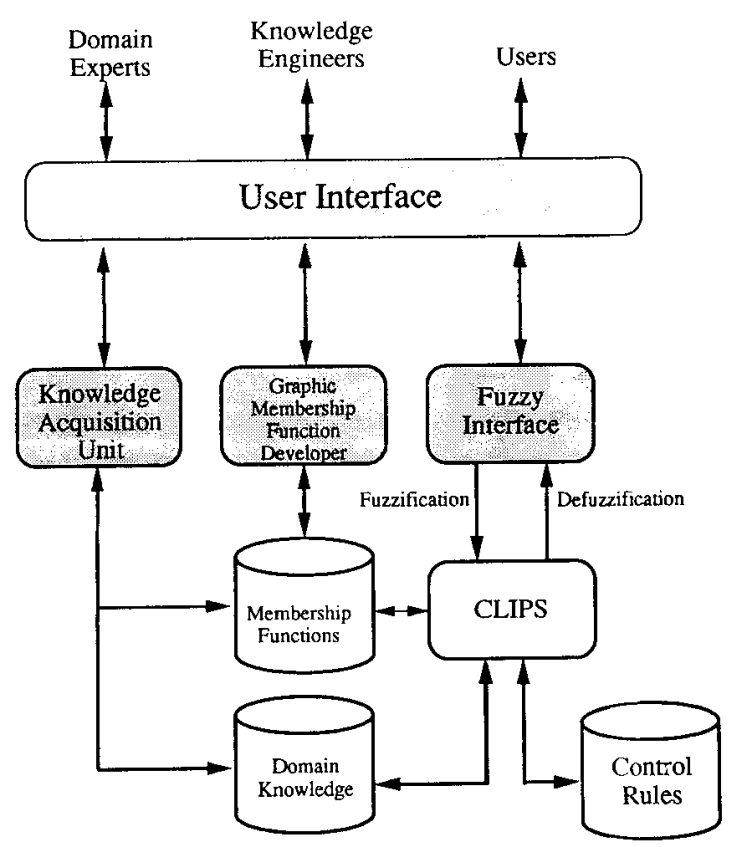

Figure 1. The structure of KAFES.

(3) Fuzzy interface for expert system shells: the interface performs the fuzzification and defuzzification operations, which facilitate fuzzy reasoning on conventional expert system shells. Currently, it is available for CLIPS, a shell for the development and delivery of expert systems developed by the Software Technology Branch of the Information System Directorate at NASA/Johnson Space Center.

(4) A set of control rules for CLIPS, which enables fuzzy reasoning on CLIPS.

The whole structure of the environment is depicted in Figure 1.

\section{A. Knowledge Acquisition Unit}

Many existing systems employ the repertory grid test, which was originally invented and widely used by George Kelly in clinical psychology ${ }^{16}$ when interviewing experts. In a repertory grid, a set of elements is listed across the top of the grid. Various combinations of three elements are then presented to the expert, and the question, "Think of an important trait or characteristic, which makes two of the elements different from the third," is asked. The trait given by the expert is listed on the left-hand side of the grid, and its opposite is listed on the right-hand side. Each pair of a trait and its opposite is called a construct. After the set of constructs is ready, the expert is asked to fill the grid with ratings. A $1 \rightarrow 5$ rating mechanism is often used in filling the grid; i.e., each rating is an integer ranging from $1-5$. A 1 is at the left-hand pole, a 5 is at the right-hand pole, while the rest fall between the poles. An example of a repertory 


\begin{tabular}{|c|ccccc|c|}
\hline & $E_{1}$ & $E_{2}$ & $E_{3}$ & $E_{4}$ & $E_{5}$ & \\
\hline$C_{1}$ & 5 & 1 & 5 & 1 & 1 & $C_{1}^{\prime}$ \\
$C_{2}$ & 4 & 4 & 4 & 1 & 4 & $C_{2}^{\prime}$ \\
$C_{3}$ & 1 & 4 & 5 & 1 & 4 & $C_{3}^{\prime}$ \\
$C_{4}$ & 1 & 1 & 1 & 5 & 1 & $C_{4}^{\prime}$ \\
\hline
\end{tabular}

Figure 2. An example of a repertory grid.

grid with five elements $\left(E_{1}, E_{2}, E_{3}, E_{4}\right.$, and $\left.E_{5}\right)$ and four constructs $\left(C_{1}, C_{2}, C_{3}\right.$, and $C_{4}$ ) is given in Figure 2.

Each rating expresses the relationship between a construct and an element, which does not provide sufficient information for building fuzzy expert systems. Consider the following repertory grid:

\begin{tabular}{ccccc}
\hline & $\begin{array}{c}\text { Dangue } \\
\text { fever }\end{array}$ & Measles & $\ldots$ & \\
\hline Low & 5 & 2 & $\ldots$ & High \\
$\cdot$ & $\cdot$ &. & $\ldots$ & $\cdot$ \\
$\cdot$ & $\cdot$ &. & $\ldots$ & $\cdot$ \\
. &. &. & $\ldots$ &. \\
\hline
\end{tabular}

From the ratings of the grid, we are only aware of the information, "For Dangue Fever, a patient is likely to have a high temperature," and, "For Measles, a patient's temperature is usually not high." It is quite possible that the expert wants to emphasize the expressions by adding some linguistic hedges:

"For Dangue Fever, a patient's temperature should be VERY high."

"For Measles, a patient's temperature is usually MORE OR LESS low."

From the examples, we find it difficult to apply conventional repertory grids to the development of fuzzy expert systems, which is due to the following reasons:

(1) Repertory grids do not explicitly hold information for linguistic hedges.

(2) Repertory grids do not hold information for helping the determination of membership functions.

Thus, it would be more desirable to adapt the grid so that it is appreciate for fuzzy logic instead of using fuzzy logic with repertory grids. To cope with these problems, we propose the fuzzy table to hold the information for generating fuzzy rules and membership functions. The procedure for constructing a fuzzy table is similar to that of a repertory grid, which consists of three steps shown as follows:

Step 1: Elicit all the elements (candidate solutions or actions) from the 
expert. The elements (e.g., $G_{1}, G_{2}, G_{3}, G_{4}$, and $G_{5}$ ) provided by the expert are placed across the top of the table:

\begin{tabular}{lllll}
$G_{1}$ & $G_{2}$ & $G_{3}$ & $G_{4}$ & $G_{5}$ \\
\hline
\end{tabular}

Step 2: Elicit attributes (fuzzy variables) from the expert. Each time, the expert is asked for an attribute so that three elements can be partially or fully distinguished. The fuzzy variables are listed on the left-hand side of the table. For each fuzzy variable, three fuzzy values are specified and listed on the righthand side according to the following procedure:

KAFES: Please select a set of fuzzy values for fuzzy variable $V_{1}$ : 1 . LOW/ MIDDLE/HIGH. 2. SHORT/MIDDLE/TALL. 3. LIGHT/NORMAL/ HEAVY. 4. SMALL/MIDDLE/BIG. 0, Other (user-defined).

EXPERT: 1 .

KAFES: Please select a set of fuzzy values for fuzzy variable $V_{2}: 1$. LOW/ MIDDLE/HIGH. 2. SHORT/MIDDLE/TALL. 3. LIGHT/NORMAL/ HEAVY. 4. SMALL/MIDDLE/BIG. 0. Other (user-defined).

EXPERT: 2.

KAFES: Please select a set of fuzzy values for fuzzy variable $V_{3}: 1$. LOW/ MIDDLE/HIGH. 2. SHORT/MIDDLE/TALL. 3. LIGHT/NORMAL/ HEAVY. 4. SMALL/MIDDLE/BIG. 0. Other (user-defined).

EXPERT: 0 .

KAFES: Please indicate the lower bound of the fuzzy values.

EXPERT: SLOW.

KAFES: Please indicate the middle of the fuzzy values.

EXPERT: NORMAL.

KAFES: Please indicate the upper bound of the fuzzy values.

EXPERT: FAST.

An example of a fuzzy table with fuzzy variable and values is given as follows:

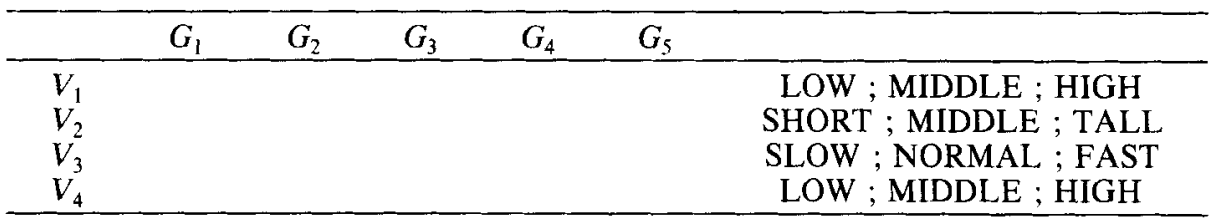

Step 3: Fill all of the [element, attribute] entries of the grid. Each entry consists of two parts: a rating to indicate the most desirable fuzzy value and the degree of certainty for giving that rating. It is possible that the expert selects the most desirable fuzzy value, but still lacks confidence about his (or her) choice.

A 7-scale rating mechanism is employed in the fuzzy table. The ratings are integers ranging from -3 to +3 , which represent the possible combinations 
of linguistic hedges and fuzzy values. Consider the ratings of fuzzy variable $V_{\text {, }}$ in the table; 3 means VERY HIGH, 2 means HIGH, 1 means MORE OR LESS HIGH, 0 means MIDDLE, -1 means MORE OR LESS LOW, -2 means LOW, and -3 means VERY LOW. The degree of certainty for each rating is described by " $S$ " or " $N$ ", which represent VERY SURE and NOT VERY SURE, respectively. For example:

\begin{tabular}{rrrrrrr}
\hline & $G_{1}$ & $G_{2}$ & \multicolumn{1}{c}{$G_{3}$} & $G_{4}$ & $G_{5}$ & \\
\hline$V_{1}$ & $3 / \mathrm{S}$ & $0 / \mathrm{N}$ & $-3 / \mathrm{S}$ & $1 / \mathrm{S}$ & $3 / \mathrm{S}$ & LOW ; MIDDLE ; HIGH \\
$V_{2}$ & $2 / \mathrm{S}$ & $1 / \mathrm{S}$ & $3 / \mathrm{S}$ & $2 / \mathrm{S}$ & $0 / \mathrm{S}$ & SHORT ; MIDDLE ; TALL \\
$V_{3}$ & $2 / \mathrm{S}$ & $2 / \mathrm{S}$ & $3 / \mathrm{S}$ & $1 / \mathrm{N}$ & $2 / \mathrm{S}$ & SLOW ; NORMAL ; FAST \\
$V_{4}$ & $-1 / \mathrm{N}$ & $2 / \mathrm{S}$ & $-2 / \mathrm{S}$ & $-3 / \mathrm{S}$ & $3 / \mathrm{S}$ & LOW ; MIDDLE ; HIGH \\
\hline
\end{tabular}

Each column of the fuzzy table will be translated to a fuzzy rule. The truth value for the output of $i-t h$ rule is computed by

$$
\text { TRUTH }=\frac{\# \text { of " } S "}{(\# \text { of " } S " \text { " } \# \text { of " } N ")} \times 0.8+0.2 .
$$

For example, the first column of the above fuzzy table is translated to the following rule:

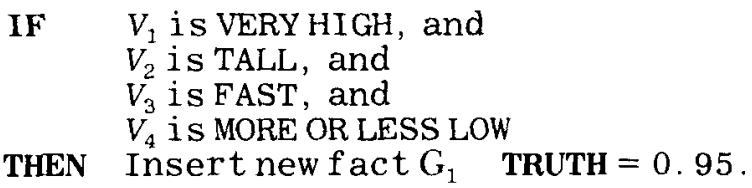

Initially, experts are asked to provide numerical information for fuzzy values, and some default functions are employed. Let fuzzy values of a fuzzy variable be represented as LS (left side), MS (middle side), and RS (right side). Assuming that the numerical values with membership degrees 1.0 for LS, MS, and RS are $\alpha, \beta$, and $\gamma$, respectively, we have the following membership functions: ${ }^{17}$

$$
\begin{gathered}
L S(x)= \begin{cases}1 & \text { for } x \leq \alpha \\
1-2\left(\frac{x-\alpha}{\gamma-\alpha}\right)^{2} & \text { for } \alpha \leq x \leq \beta \\
2\left(\frac{x-\gamma}{\gamma-\alpha}\right)^{2} & \text { for } \beta \leq x \leq \gamma \\
0 & \text { for } x \geq \gamma\end{cases} \\
M S(x)= \begin{cases}0 & \text { for } x \leq \alpha \\
2\left(\frac{x-\alpha}{\gamma-\alpha}\right)^{2} & \text { for } \alpha \leq x \leq \beta \\
1-2\left(\frac{x-\gamma}{\gamma-\alpha}\right)^{2} & \text { for } \beta \leq x \leq \gamma \\
1 & \text { for } x \geq \gamma\end{cases}
\end{gathered}
$$




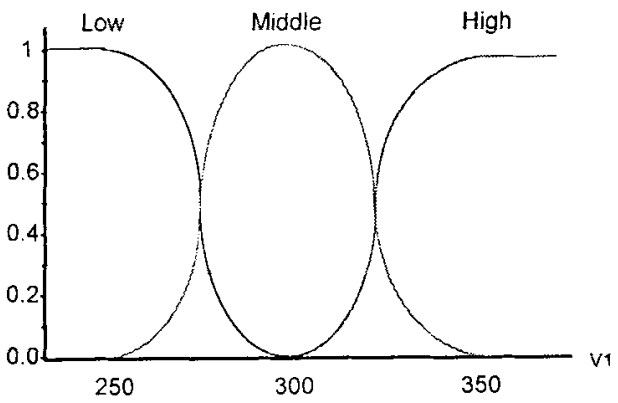

Figure 3. Graphic representation of fuzzy values for $V_{1}$.

$$
R S(x)= \begin{cases}0 & \text { for } x \leq \alpha \\ 2\left(\frac{x-\alpha}{\beta-\alpha}\right)^{2} & \text { for } \alpha \leq x \leq \frac{(\alpha+\beta)}{2} \\ 1-2\left(\frac{x-\beta}{\beta-\alpha}\right)^{2} & \text { for } \frac{(\alpha+\beta)}{2} \leq x \leq \beta \\ 1-2\left(\frac{x-\beta)}{\gamma-\beta}\right)^{2} & \text { for } \beta \leq x \leq \frac{(\beta+\gamma)}{2} \\ 2\left(\frac{x-\gamma}{\gamma-\beta}\right)^{2} & \text { for } \frac{(\beta+\gamma)}{2} \leq x \leq \gamma \\ 0 & \text { for } x \geq \gamma .\end{cases}
$$

For $V_{1}$ in the above table, if the expert gives numerical value 250 for fuzzy value LOW (LS), 300 for MIDDLE (MS), and 350 for HIGH (RS), membership functions given in Figure 3 are derived.

The degrees of memberships of linguistic hedges are computed by the following hedge functions:

$$
\begin{aligned}
& \text { 1. } \mathrm{F}(\mathrm{X})_{N O T}=1-X \\
& \text { 2. } \mathrm{F}(\mathrm{X})_{V E R Y}=X^{2} \\
& \text { 3. } \mathrm{F}(\mathrm{X})_{M O R E-O R-L E S S}=X^{0.5} \text {, }
\end{aligned}
$$

where $X$ is the degree of memberships without considering linguistic hedges.

\section{B. Graphic Membership Function Developer}

Graphic membership function developer is used to help knowledge engineers to create and adjust membership functions for input facts. It provides a graphic view, so that knowledge engineers can adjust the shape of membership function curves directly to get the desirable results. 


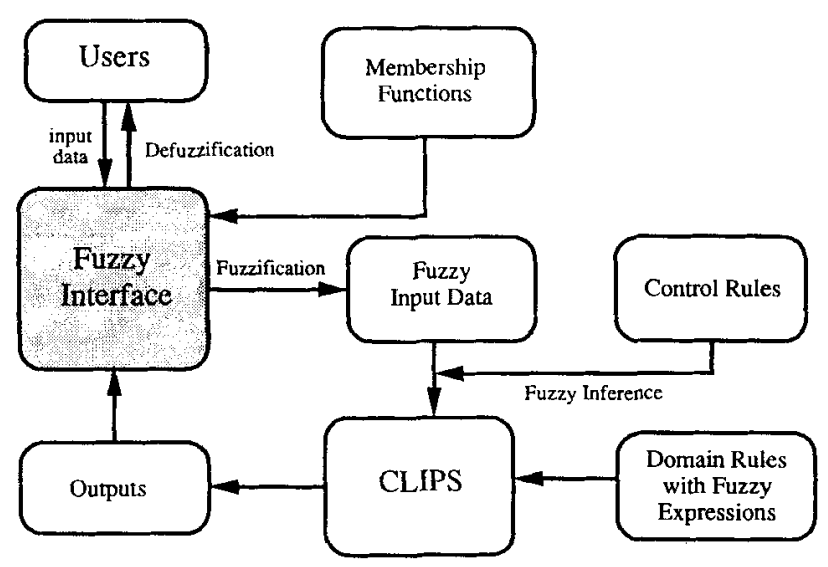

Figure 4. Fuzzy reasoning by applying the fuzzy interface.

\section{Fuzzy Interface}

The knowledge representation and inference process in a fuzzy expert system is quite different from those in a conventional expert system. However, as there already exist many expert system shells and knowledge engineering tools for conventional expert systems, it would be very convenient if we could build fuzzy expert systems based upon the existing shells or tools. We selected one of the most popular expert system shells, C Language Integrated Production System (CLIPS) to work with the knowledge acquisition system. CLIPS is a tool for the development and delivery of expert systems developed by the Software Technology Branch of the Information System Directorate at NASA/ Johnson Space Center.

The fuzzy interface is capable of accepting input values from users, performing fuzzification operations, invoking the inference of CLIPS, and performing defuzzification operations on the outputs from CLIPS. If the knowledge engineer or the expert is not satisfied with the system output, the graphic developer is helpful in adjusting the membership functions to achieve better performance. The process of fuzzy reasoning by applying the fuzzy interface is shown in Figure 4.

After performing the inference process, all the system outputs are collected by the fuzzy interface to make the final conclusion. The centroid defuzzification is employed to derive a more reasonable result, which will be shown by graphic representation as well as text display to facilitate the modification and debugging of the fuzzy expert systems.

\section{Control Rules for Fuzzy Reasoning}

A significant difference between fuzzy rules and conventional rules is the use of linguistic hedges, such as NOT, VERY, NOT VERY, MORE OR LESS, NOT MORE OR LESS, etc. For example, two rules with truth value 1.0 are given as follows: 


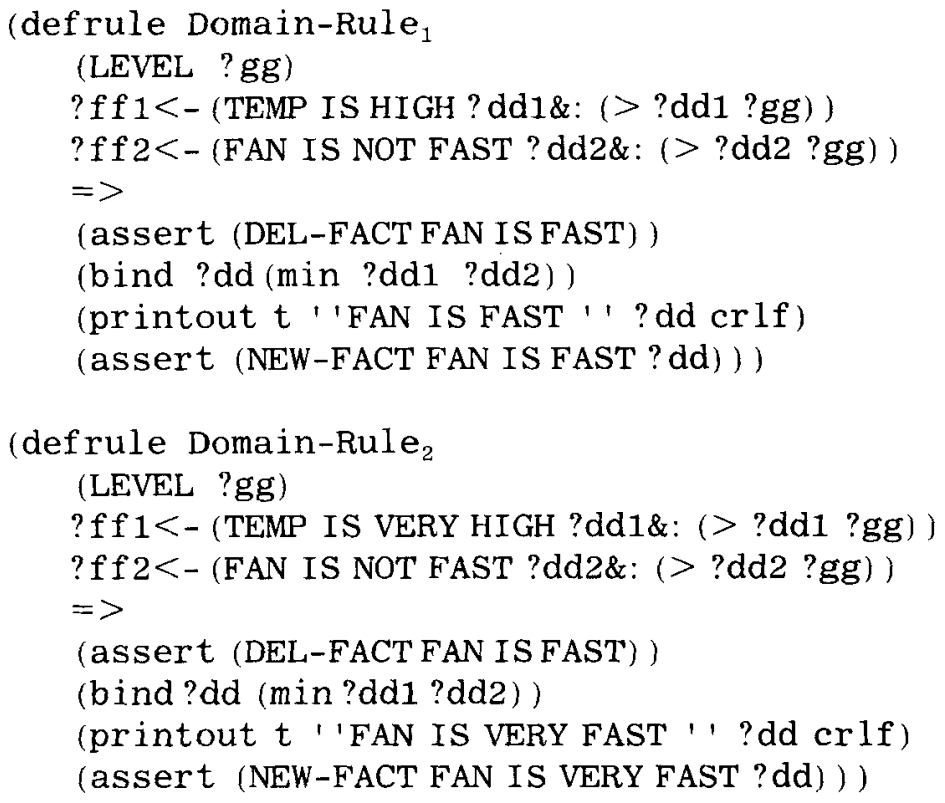

For an input value $\left(\mathrm{e} . \mathrm{g}, 2^{\circ} \mathrm{C}\right)$, the fuzzy interface first performs fuzzification operations to produce the corresponding fuzzy fact (e.g., temperature is NORMAL 0.95), and then transfers it to CLIPS for inference. The problem is, how could those fuzzy facts match the premise patterns of rules if linguistic hedges exist. For example, the fact (TEMPERATURE IS HIGH 0.6) should be able to match the patterns (TEMPERATURE IS VERY HIGH) with degree 0.36, (TEMPERATURE IS HIGH) with degree 0.6, (TEMPERATURE IS LOW) with degree 0.4 , (TEMPERATURE IS NOT VERY HIGH) with degree 6.4 , etc. To cope with this problem, some control rules are used to generate a set of facts with linguistic hedges to replace the original fuzzy fact. One such control rule is shown as follows:

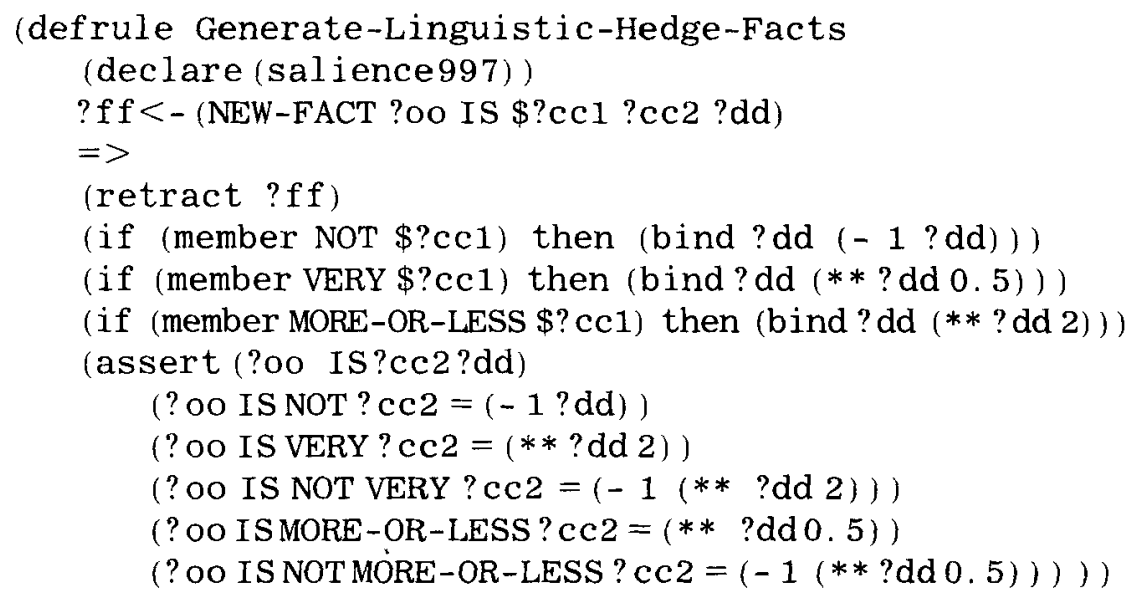


For example, let the input fact be (temperature is $18^{\circ} \mathrm{C}$ ), the following fuzzy facts will be produced by fuzzification operations:

(temperature is HIGH 0.5 )

(temperature is MIDDLE 0.8)

(temperature is LOW 0.0)

The fact (temperature is LOW 0.0) is not removed immediately, since some of its corresponding facts with linguistic hedges will have nonzero degrees. To enable fuzzy inference with linguistic hedges, the following fuzzy facts are generated by the control rules to replace the three original facts:

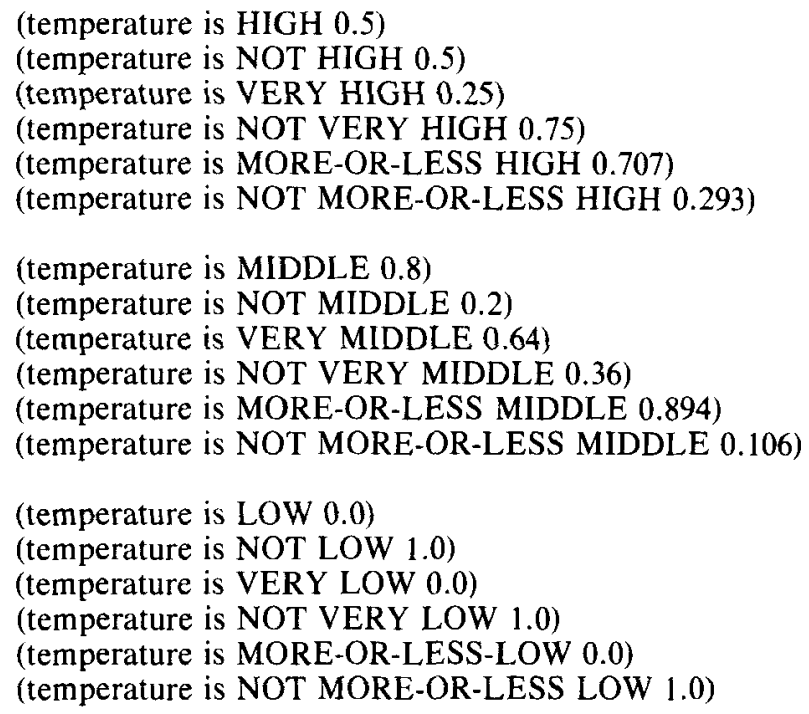

\section{EVALUATION OF THE NEW APPROACH}

Some experiments concerning the diagnosis of Acute Exanthema were made to evaluate the new approach. The earlier knowledge base was constructed by applying the EMCUD method, ${ }^{18}$ and 47 rules are generated for 14 diseases. In Appendix A, it can be seen that there are lots of redundant rules although the test results (in Table I) seem to be desirable. With the new approach, 14 fuzzy rules are generated (as given in Appendix B) with the same test results.

\section{CONCLUSIONS}

In this article, we propose a knowledge acquisition system, KAFES, for constructing fuzzy expert systems. We have applied KAFES to a medical application. According to our experience, it can be seen that the use of fuzzy concepts to represent expertise significantly reduces the redundant rules in the knowledge bases while achieving a promising performance. Moreover, it seems 
Table I. Test results of the knowledge base with redundant rules. ${ }^{\text {a }}$

\begin{tabular}{lrrrrrrrrrrrrr}
\hline Case number & 1 & 2 & 3 & 4 & 5 & 6 & 7 & 8 & 9 & 10 & 11 & 12 & 13 \\
Physician & 12 & 3 & 3 & 1 & 2 & 1 & 14 & 2 & 6 & 5 & 5 & 3 & 1 \\
Without redundant rules & 12 & $\mathrm{X}$ & $\mathrm{X}$ & $\mathrm{X}$ & $\mathrm{X}$ & $\mathrm{X}$ & 14 & $\mathrm{X}$ & 6 & $\mathrm{X}$ & $\mathrm{X}$ & 3 & 1 \\
With redundant rules & 12 & 3 & 3 & 1 & 2 & 1 & 14 & 2 & 6 & 5 & 5 & 3 & 1 \\
& & & & & & & & & & & & \\
Case number & 14 & 15 & 16 & 17 & 18 & 19 & 20 & 21 & 22 & 23 & 24 & 25 \\
Physician & 6 & 6 & 12 & 5 & 8 & 9 & 14 & 13 & 4 & 1 & 2 & 14 \\
Without redundant rules & $\mathrm{X}$ & $\mathrm{X}$ & 12 & 5 & $\mathrm{X}$ & 9 & 14 & 13 & 4 & 1 & 2 & 14 \\
With redundant rules & 6 & 6 & 12 & 5 & 8 & 9 & 14 & 13 & 4 & 1 & 2 & 14 \\
\hline
\end{tabular}

${ }^{a}$ The codes of diseases and their translations are: 1-Measles, 2-German measles, 3-Chickenpox, 4-Smallpox, 5-Scarlet fever, 6-Exanthem subitum, 7-Fifth disease, 8-Meningococcemia, 9-Rocky Mt. spotted fever, 10-Typhus fevers, 11-Infectious mononucleosis, 12-Enterovirus infections, 13-Drug eruptions, and 14-Eczema herpeticum.

to be more natural and straightforward to represent knowledge of experts by fuzzy rules. Currently, we are trying to enhance the system by applying multimedia techniques. Some related studies, including the learning algorithm for the adjustment of membership functions, the improvement of inference methodologies, and the representation of multimedia knowledge bases, are in progress.

\section{APPENDIX A: THE REPERTORY GRID FOR ACUTE EXANTHEMA}

$\begin{array}{ll}D_{1} \text { - Measles } & D_{8} \text { - Meningococcemia } \\ D_{2} \text { - German measles } & D_{9} \text { - Rocky Mt. spotted fever } \\ D_{3} \text { - Chickenpox } & D_{10} \text { - Typhus fevers } \\ D_{4} \text { - Smallpox } & D_{11} \text { - Infectious mononucleosis } \\ D_{5} \text { - Scarlet } & D_{12} \text { - Enterovirus infections } \\ D_{6} \text { - Exanthem subitum } & D_{13} \text { - Drug eruptions } \\ D_{7} \text { - Fifth disease } & D_{14} \text { - Eczema herpeticum }\end{array}$

\begin{tabular}{lcccccccl}
\hline & $D_{1}$ & $D_{2}$ & $D_{3}$ & $D_{4}$ & $D_{5}$ & $D_{6}$ & $D_{7}$ & \\
\hline Koplik's spots & 1 & 3 & 3 & 3 & 3 & 3 & 3 & No Koplik's spots \\
3-4 days of fever & 1 & 5 & 3 & 3 & 4 & 1 & 5 & Not 3-4 days \\
1-2 days of fever & 3 & 5 & 4 & 4 & 2 & 3 & 5 & Not 1-2 days \\
Headache & 3 & 3 & 1 & 1 & 3 & 3 & 3 & No headache \\
Flushed cheeks & 3 & 3 & 3 & 3 & 3 & 3 & 1 & No flushed cheeks \\
Vomiting & 5 & 5 & 5 & 5 & 1 & 5 & 5 & No vomiting \\
Lymphadenopathy & 3 & 1 & 3 & 3 & 3 & 3 & 3 & No Lymphadenopathy \\
Red Maculopapular & 1 & 2 & 2 & 2 & 1 & 2 & 1 & Not red \\
Deep lesion & 3 & 3 & 5 & 1 & 3 & 3 & 3 & Superficial \\
Centripetal & 3 & 3 & 1 & 5 & 3 & 3 & 3 & Centrifugal \\
Circumoral pallor & 5 & 5 & 5 & 5 & 1 & 5 & 1 & No circumoral pallor \\
Petechiae & 5 & 5 & 5 & 3 & 5 & 5 & 5 & No petechiae \\
Splenomegaly & 3 & 3 & 3 & 3 & 3 & 3 & 3 & No Splenomegaly \\
Chills & 3 & 5 & 3 & 1 & 3 & 3 & 5 & No chills \\
Sore throat & 3 & 3 & 3 & 1 & 3 & 3 & 3 & No sore throat \\
Desquamation & 1 & 5 & 3 & 3 & 1 & 3 & 3 & No desquamation \\
\hline
\end{tabular}




\begin{tabular}{lcccccccl}
\hline & $D_{8}$ & $D_{9}$ & $D_{10}$ & $D_{11}$ & $D_{12}$ & $D_{13}$ & $D_{14}$ & \\
\hline Koplik's spots & 3 & 3 & 3 & 3 & 3 & 3 & 3 & No Koplik's spots \\
3-4 days of fever & 4 & 1 & 1 & 4 & 4 & 4 & 5 & Not 3-4 days \\
1-2 days of fever & 2 & 4 & 2 & 2 & 1 & 2 & 5 & Not 1-2 days \\
Headache & 3 & 1 & 1 & 3 & 3 & 3 & 3 & No headache \\
Flushed cheeks & 3 & 3 & 3 & 3 & 3 & 3 & 3 & No flushed cheeks \\
Vomiting & 1 & 5 & 5 & 5 & 5 & 5 & 5 & No vomiting \\
Lymphadenopathy & 3 & 3 & 3 & 3 & 3 & 3 & 3 & No Lymphadenopathy \\
Red Maculopapular & 2 & 2 & 2 & 2 & 2 & 2 & 2 & Not red \\
Deep lesion & 3 & 3 & 3 & 3 & 3 & 3 & 3 & Superficial \\
Centripetal & 3 & 5 & 1 & 3 & 3 & 3 & 3 & Centrifugal \\
Circum. pallor & 5 & 5 & 5 & 5 & 5 & 5 & 5 & No circumoral pallor \\
Petechiae & 1 & 1 & 1 & 3 & 5 & 5 & 3 & No petechiae \\
Splenomegaly & 3 & 3 & 3 & 1 & 3 & 3 & 3 & No Splenomegaly \\
Chills & 3 & 1 & 1 & 3 & 3 & 3 & 5 & No chills \\
Sore throat & 3 & 3 & 3 & 1 & 3 & 3 & 5 & No sore throat \\
Desquamation & 3 & 3 & 3 & 3 & 3 & 3 & 3 & No desquamation \\
\hline
\end{tabular}

Rules generated from the Repertory Grids by EMCUD:

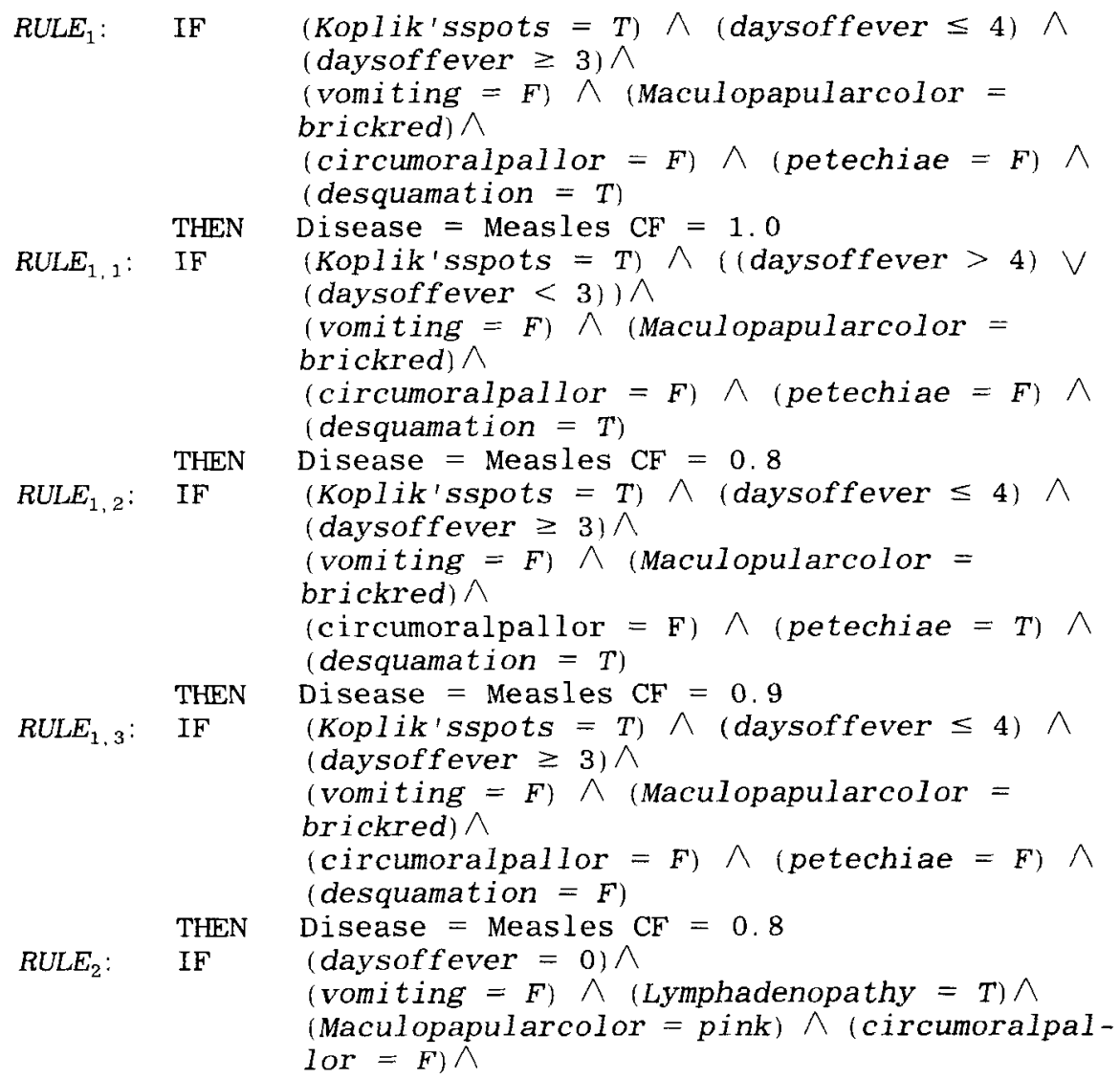









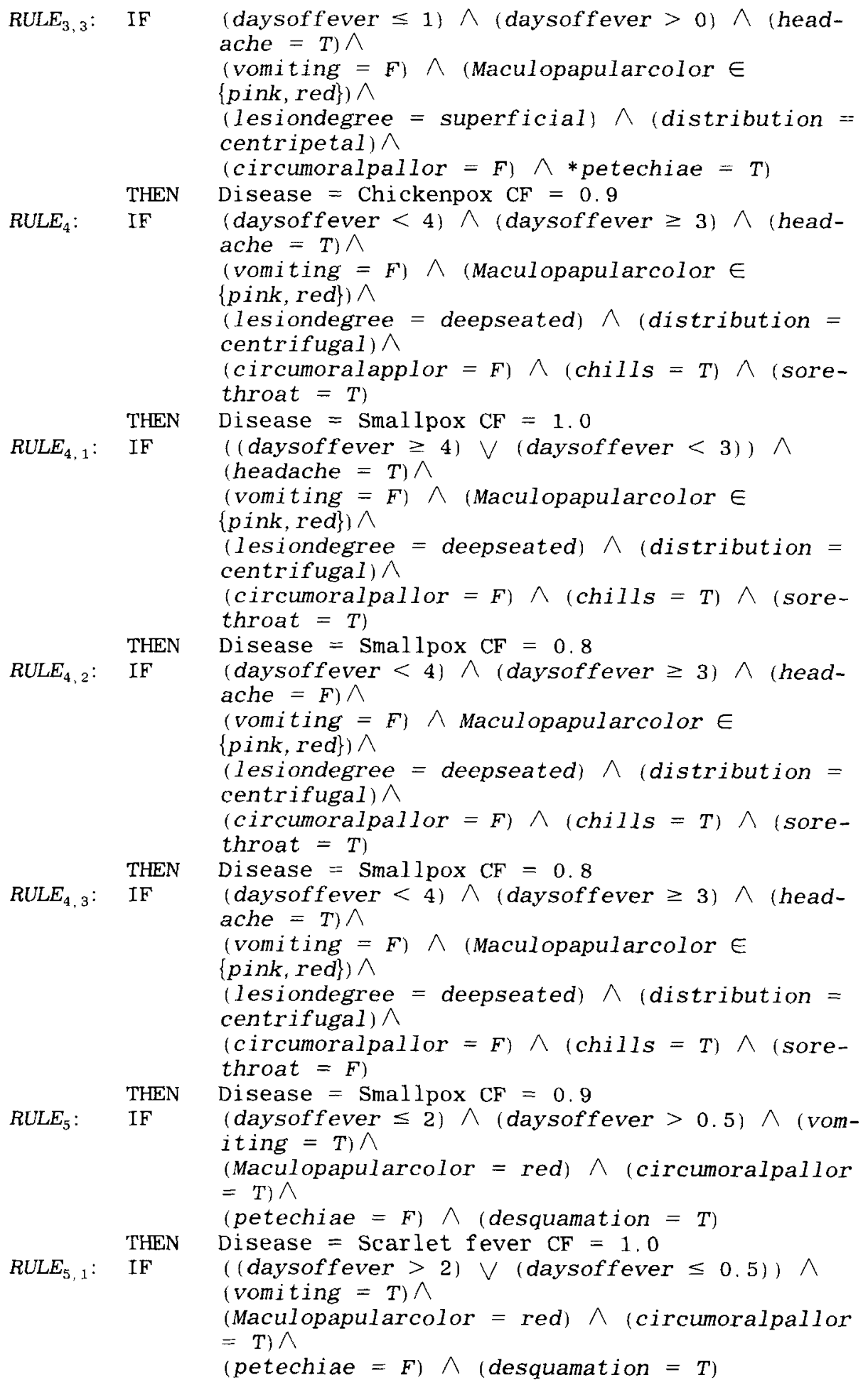


THEN Disease $=$ Scarlet fever $\mathrm{CF}=0.8$

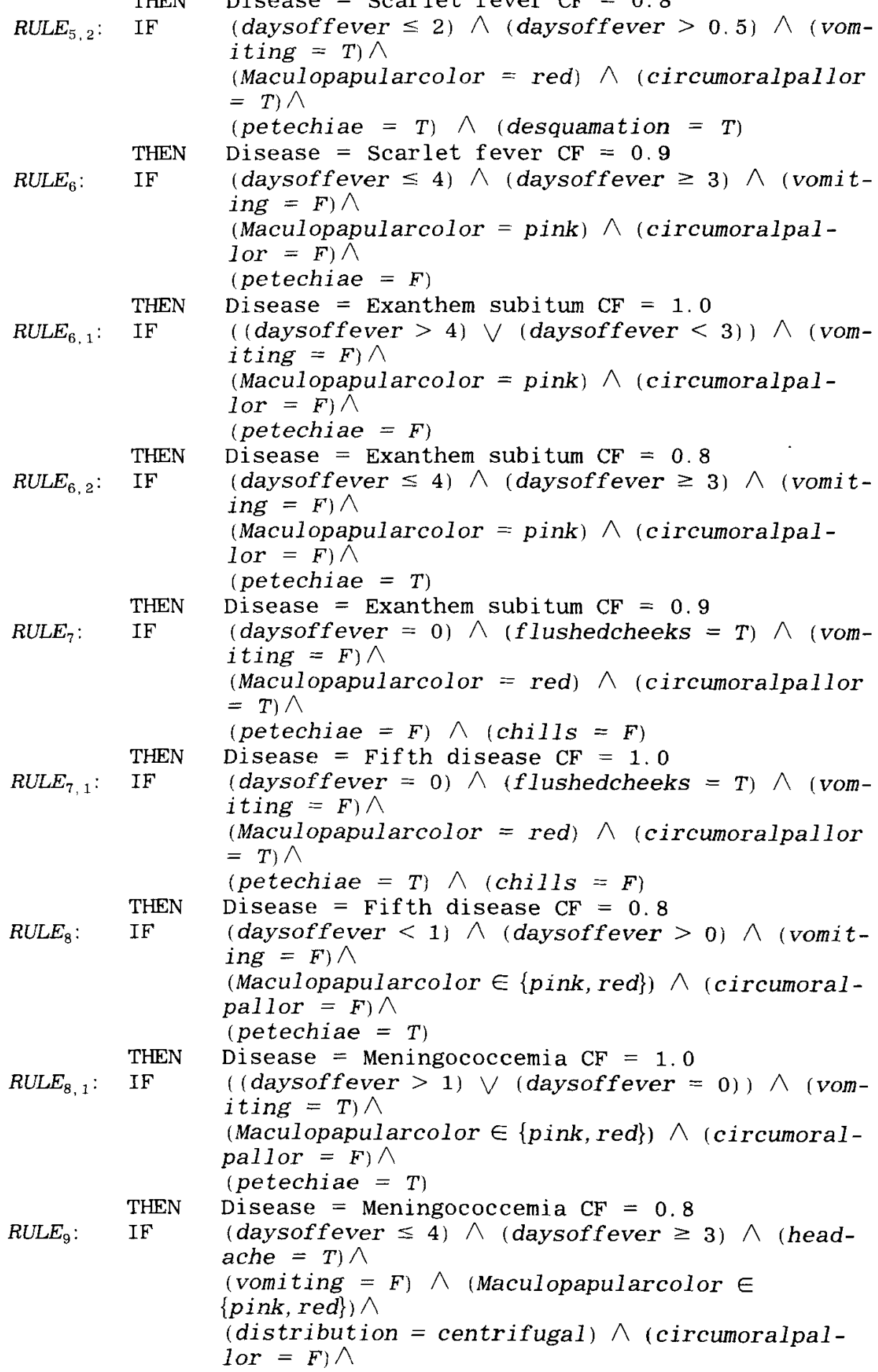




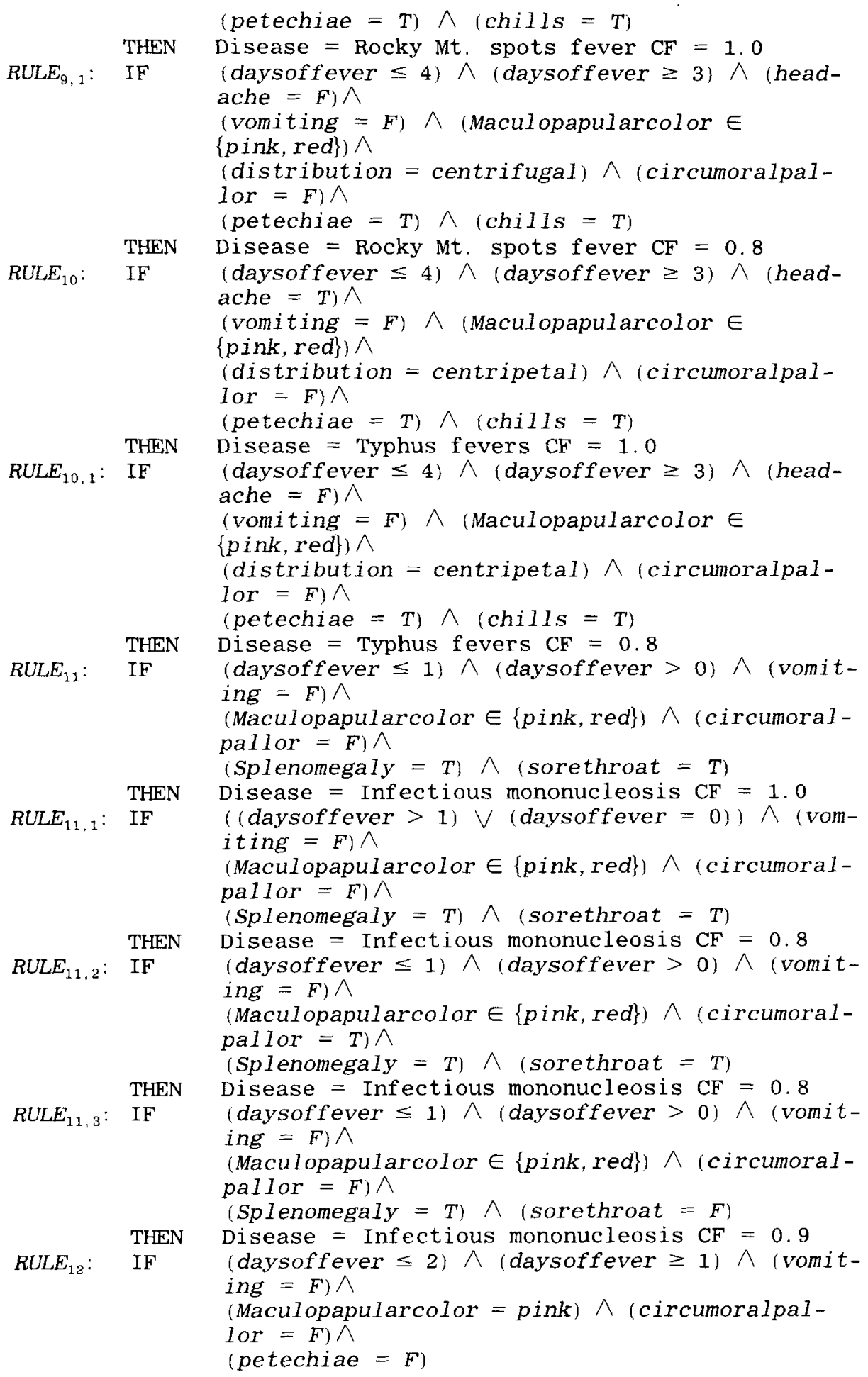




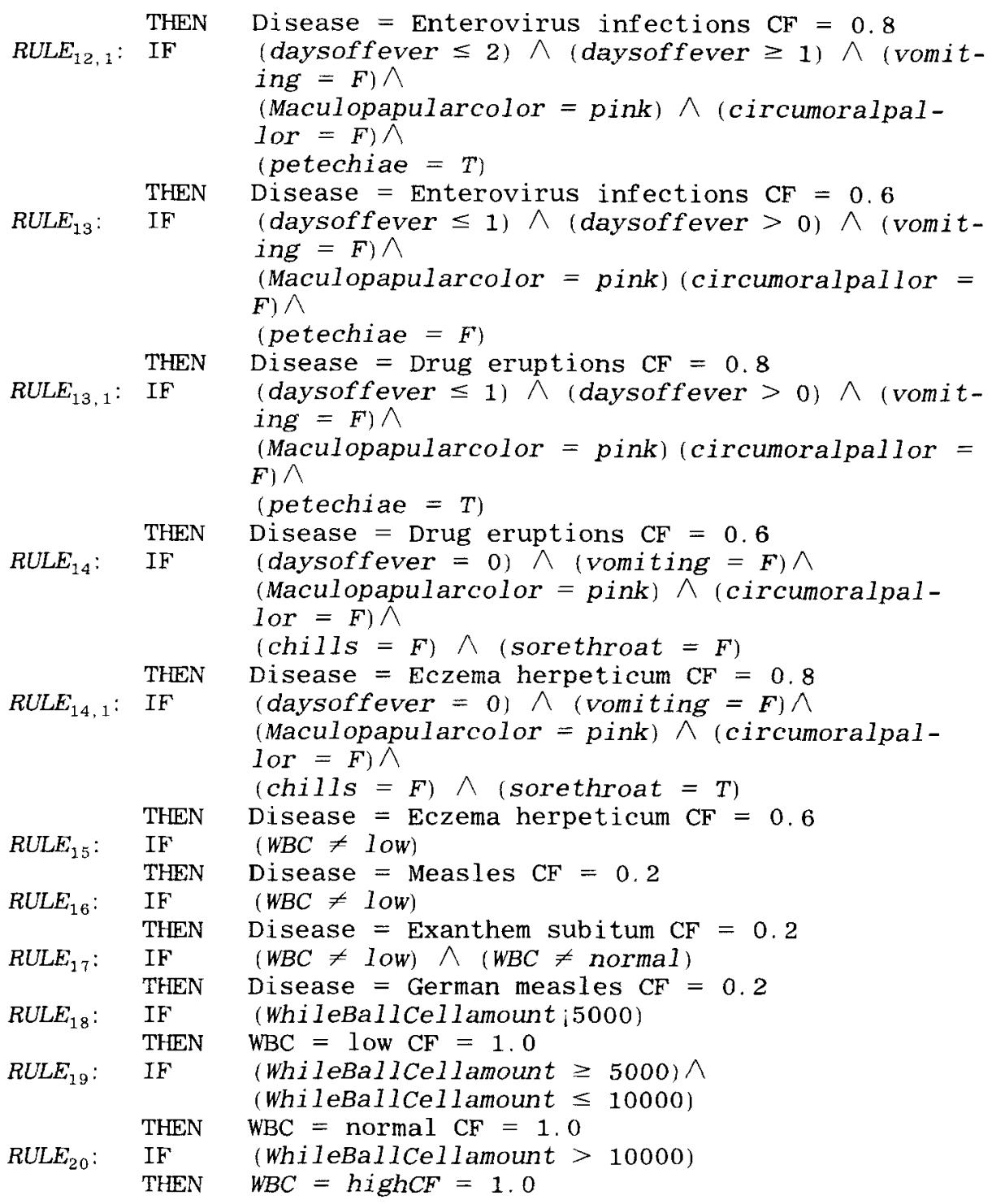

\section{APPENDIX B: FUZZY RULES}

RULE $E_{1}$ : IF (Koplik's spots is definite) ^ (fever is serious) $\wedge$ (vomiting is not apparent) $\wedge$ (Maculopapular color is brick red) $\wedge$ (circumoral pallor is not apparent) $\wedge$ (petechiae is not apparent) $\wedge$ (desquamation is apparent)

THEN Disease $=$ Measles $\mathrm{CF}=1.0$

RULE $E_{2}: \quad$ IF (fever is none) $\wedge$ (petechiae is not apparent) $\wedge$ (vomiting is not apparent) $\wedge$ (Lymphadenopathy is definite) $\wedge$ 


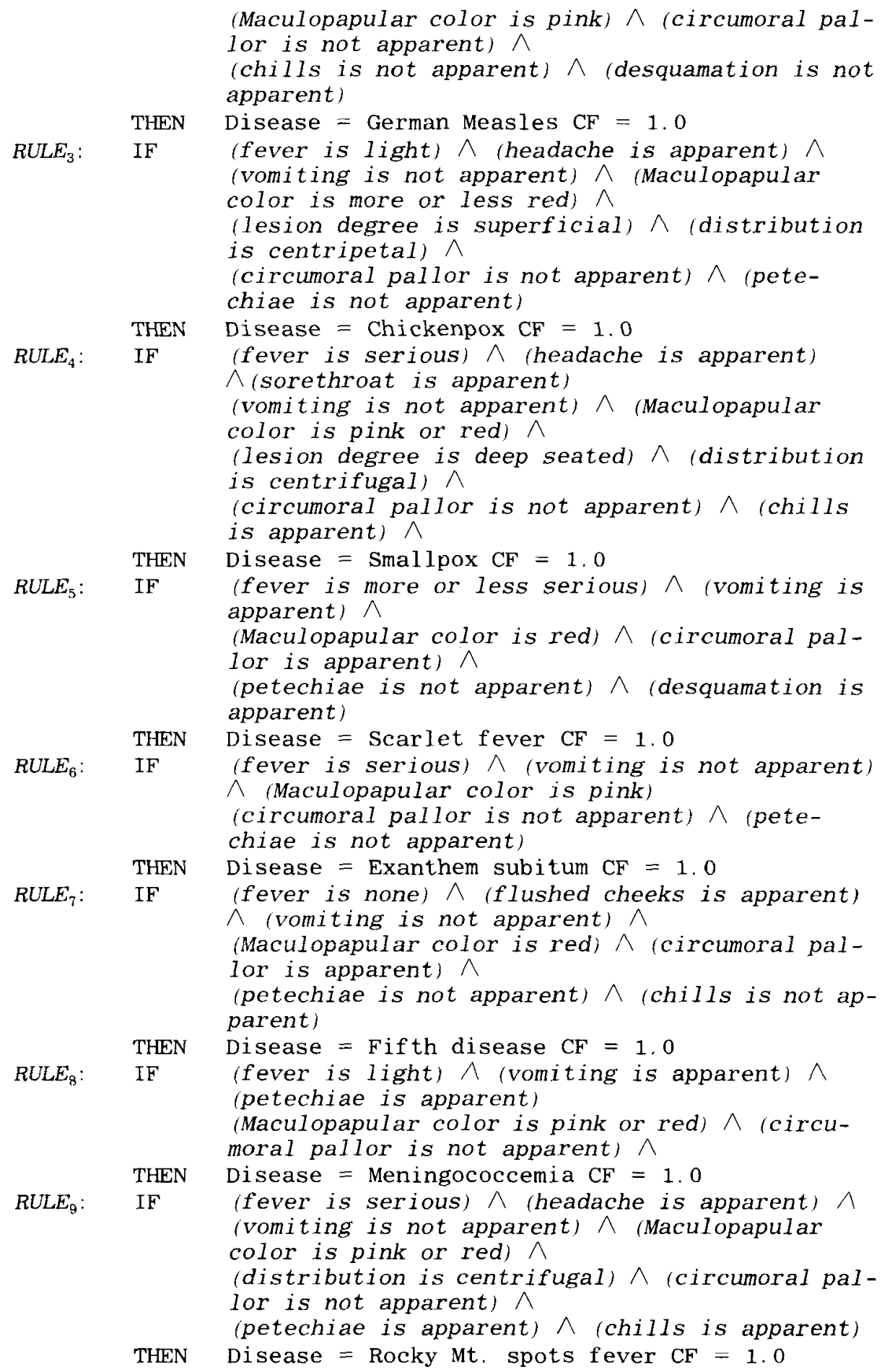

(Maculopapular color is pink) $\wedge$ (circumoral pallor is not apparent) $\wedge$

(chills is not apparent) $\wedge$ (desquamation is not apparent)

THEN Disease $=$ German Measles $\mathrm{CF}=1.0$

RULE $_{3}:$ IF (fever is light) $\wedge$ (headache is apparent) $\wedge$

(vomiting is not apparent) $\wedge$ (Maculopapular color is more or less red) $\wedge$

(lesion degree is superficial) $\wedge$ (distribution is centripetal) $\wedge$

(circumoral pallor is not apparent) $\wedge$ (petechiae is not apparent)

THEN Disease $=$ Chickenpox $\mathrm{CF}=1.0$

RULE $E_{4}$ IF (fever is serious) $\wedge$ (headache is apparent)

$\wedge$ (sorethroat is apparent)

(vomiting is not apparent) $\wedge$ (Maculopapular color is pink or red) $\wedge$

(lesion degree is deep seated) $\wedge$ (distribution is centrifugal) $\wedge$

(circumoral pallor is not apparent) $\wedge$ (chills

is apparent) $\wedge$

THEN Disease $=$ Smallpox $\mathrm{CF}=1.0$

RULE $E_{5}$ IF (fever is more or less serious) $\wedge$ (vomiting is apparent) $\wedge$

(Maculopapular color is red) $\wedge$ (circumoral pallor is apparent) $\wedge$

(petechiae is not apparent) $\wedge$ (desquamation is apparent)

THEN Disease $=$ Scarlet fever $\mathrm{CF}=1.0$

$R U L E_{6}:$ IF (fever is serious) $\wedge$ (vomiting is not apparent)

$\wedge$ (Maculopapular color is pink)

(circumoral pallor is not apparent) $\wedge$ (pete-

chiae is not apparent)

THEN Disease $=$ Exanthem subitum $\mathrm{CF}=1.0$

$R U L E_{7}:$ IF (fever is none) $\wedge$ (flushed cheeks is apparent)

$\wedge$ (vomiting is not apparent) $\wedge$

(Maculopapular color is red) $\wedge$ (circumoral pallor is apparent) $\wedge$

(petechiae is not apparent) $\wedge$ (chills is not apparent)

THEN Disease $=$ Fif th disease $C F=1.0$

RULE $E_{8}$ IF (fever is light) $\wedge$ (vomiting is apparent) $\wedge$ (petechiae is apparent)

(Maculopapular color is pink or red) $\wedge$ (circumoral pallor is not apparent) $\wedge$

THEN Disease $=$ Meningococcemia $\mathrm{CF}=1.0$

RULE $E_{9}$ IF (fever is serious) $\wedge$ (headache is apparent) $\wedge$ (vomiting is not apparent) $\wedge$ (Maculopapular color is pink or red) $\wedge$

(distribution is centrifugal) $\wedge$ (circumoral pallor is not apparent) $\wedge$

(petechiae is apparent) $\wedge$ (chills is apparent)

THEN Disease $=$ Rocky Mt. spots fever $\mathrm{CF}=1.0$ 


\begin{tabular}{|c|c|c|}
\hline \multirow[t]{2}{*}{$R U L E_{10}:$} & IF & $\begin{array}{l}\text { (fever is serious) } \wedge \text { (headahce is apparent) } \wedge \\
\text { (vomiting is not apparent) } \wedge \text { (Maculopapular } \\
\text { color is pink or red) } \\
\text { (distribution is centripetal) } \wedge \text { (circumoral pal- } \\
\text { lor is not apparent) } \wedge \\
\text { (petechiae is apparent) } \wedge \text { (chills is apparent) }\end{array}$ \\
\hline & THEN & Disease $=$ Typhus fevers $\mathrm{CF}=1.0$ \\
\hline \multirow[t]{2}{*}{$R U L E_{11}:$} & IF & $\begin{array}{l}(\text { fever is light) } \wedge \text { (vomiting is not apparent) } \\
\wedge \text { (Maculopapular color is pink or red) } \wedge \text { (cir- } \\
\text { cumoral pallor is not apparent) } \wedge \\
\text { (Splenomegaly is apparent) } \wedge \text { (sorethroat is ap- } \\
\text { parent) }\end{array}$ \\
\hline & THEN & Disease $=$ Infectious mononucleosis $\mathrm{CF}=1.0$ \\
\hline \multirow[t]{2}{*}{$R U L E_{12}$ : } & IF & $\begin{array}{l}\text { (fever is more or less serious) } \wedge \text { (vomiting is } \\
\text { not apparent) } \wedge \\
\text { (Maculopapular color is more or less pink) } \wedge \\
\text { (circumoral pallor is not apparent) } \\
\text { (petechiae is not apparent) }\end{array}$ \\
\hline & THEN & Disease $=$ Enterovirus infections $\mathrm{Cf}=0.8$ \\
\hline \multirow[t]{2}{*}{$R U L E_{13}$ : } & IF & $\begin{array}{l}(\text { fever is light) } \wedge \text { (vomiting is not apparent) } \\
\wedge \text { (petechiae is not apparent) } \\
\text { (Maculopapular color is more or less pink) (cir- } \\
\text { cumoral pallor is not apparent) / }\end{array}$ \\
\hline & THEN & Disease $=$ Drug eruptions $\mathrm{CF}=0.8$ \\
\hline \multirow[t]{2}{*}{$R U L E_{14}:$} & IF & $\begin{array}{l}\text { (fever is none) } \wedge \text { (vomiting is not apparent) } \wedge \\
\text { (Maculopapular color is more or less pink) } \wedge \\
\text { (circumoral pallor is not apparent) } \\
\text { (chills is not apparent) } \wedge \text { (sorethroat is not } \\
\text { apparent) }\end{array}$ \\
\hline & & Disease $=$ Eczema herpeticum $C F=0.8$ \\
\hline
\end{tabular}

This study was supported by the National Science Council, Republic of China, under the contract number: NSC82-0408-E-009-049.

\section{References}

1. G.J. Klir and T.A. Folger, Fuzzy Sets, Uncertainty, and Information, Prentice-Hall, New Jersey 1988.

2. J. Boose, "A knowledge acquisition program for expert systems based on personal construct psychology," Int. J. Man-Mach. Stu., 23, 495-525.

3. J. Boose and J.M. Bradshaw, "NeoETS: capturing expert system knowledge in hierarchical rating grids," IEEE Expert System in Government Symposium, 1986.

4. J. Boose and J.M. Bradshaw, "Expertise transfer and complex problems: using AQUINAS as a knowledge acquisition workbench for knowledge-based systems," Int. J. Man-Mach. Stu., 26, 3-28 (1987).

5. L. Esheman, D. Ehret, J. McDermott, and M. Tan, "MOLE: a tenacious knowledge acquisition tool," Int. J. Man-Mach. Stu., 26, 41-54 (1987).

6. K.M. Ford, F.E. Petry, J.R. Adams-Webber, and P.J. Chang, "An approach to knowledge acquisition based on the personal construct systems," IEEE Trans. Knowl. and Data Eng., 3(1), 78-88 (1991).

7. K.M. Ford, A. Canas, J. Jones, H. Stahl, J. Novak, and J.R. Adams-Webber, 
"ICONKAT: an integrated constructivist knowledge acquisition tool," Int. J. Knowl. Acq., 3, 215-236 (1991).

8. B.R. Gaines, "An overview of knowledge acquisition and transfer," Int. J. Man-Mach. Stu., 26, 453-472 (1987).

9. S.T. Gruber, The Acquisition of Strategic Knowledge, Academic Press Inc., San Diego, 1988.

10. G. Kahn, S. Nowlan, and J. McDermott, "MORE: an intelligent knowledge acquisition tool," International Joint Conference of Artificial Intelligence, 31, 495-515 (1985).

11. S. Marcus, "Taking backtracking with a grain of SALT," Int. J. Man-Mach. Stu., 26, 383-398 (1987).

12. R.M. O'Bannon, "An intelligent aid to assist knowledge engineers with interviewing experts," IEEE Western Conference on Expert System, 1987.

13. M.L.G. Shaw and B.R. Gaines, "KITTEN: knowledge initiation and transfer tools for experts and novices," Int. J. Man-Mach. Stud., 27, 251-280 (1987).

14. J.M. Bradshaw, K.M. Ford, J.R. Adams-Webber, and J.H. Boose, "Beyond the repertory grid: new approaches to constructivist knowledge acquisition tool development," Int. J. Intell. Sys., 8, 287-333 (1993).

15. L.E. Wood and J.M. Ford, "Structuring interviewings with experts during knowledge elicitation," Int. J. Intel. Sys., 8, 71-90 (1993).

16. G.A. Kelly, The Psychology of Personal Constructs, Norton, New York, 1955.

17. J. Giarratano and G. Riley, Expert Systems: Principles and Programming, PWSKENT Publishing Company, Boston, 1989.

18. G.J. Hwang and S.S. Tseng, "EMCUD: a knowledge acquisition method which captures embedded meanings under uncertainty," Int. I. Man-Mach. Stu., 33, 431-451 (1990). 